good genes or some other resource from the father.

There is some evidence, however, that females may have 'pre-existing preferences' for particular traits, and that males may evolve merely to exploit females' sensory biases ${ }^{1-3}$. Female frogs of the species Physalaemus coloradorum prefer the 'whine and chuck' calls of males from the sister species $P$. pustulosus to the 'whine' only calls of males of their own species (M. Ryan, University of Texas, Austin), even though they will not have heard them before. Phylogenetic studies suggest that the preference is ancestral to both species. It is significant that the hidden preference is for a qualitatively different call; a preference for a supernormal or exaggerated form of the call that they usually hear would be expected on theoretical grounds from the runaway, good genes, or better resources views of sexual selection.

Lucky will be the first $P$. coloradorum male to produce a call with chucks: theoretical models show that this trait could spread through the population even without any coevolutionary change in female preference 4 . Whether 'preexisting bias' represents a new explanation for sexually selected traits or a special case of existing models is controversial. In $P$. pustulosus the larger males produce calls with chucks of lower frequency. Females not only prefer these calls, but have more of their eggs fertilized when they mate with the larger males ${ }^{1}$.

Research that assigns genetic algorithms the task of learning discrimination tasks suggests that all perceptual systems have inherent and unpredictable hidden biases for stimuli that they have never seen (A. Arak, Archway Engineering, UK). To illustrate this point, consider an insect pollinator that has only two plant species in its environment, $\mathrm{A}$ and $\mathrm{B}$, and prefers species A to species B. Flowers of species $\mathrm{A}$ have long petals, those of $\mathrm{B}$ are short, and the insect discriminates between the two flowers solely on this basis. Perfect discrimination would result if the insect's sensory system responded to $\mathrm{A}$ and $\mathrm{B}$ flowers with a step function: very low levels to B flowers, very high levels to A flowers, and very low levels to points in between. But an inverted ' $U$ ' shaped function that was low at B, higher at $\mathrm{A}$, but highest in between would discriminate equally well between $\mathrm{A}$ and $\mathrm{B}$, but would contain an inherent and never-tested sensory bias for flowers of intermediate size.

In principle, an infinite number of discrimination functions that yield hidden biases can be constructed. Such biases, if widespread in nature, would circumscribe the notion of an evolutionarily stable strategy (ESS) ${ }^{5}$ when applied to discrimination (Arak). All ESS

\title{
Decameric drug sandwich
}

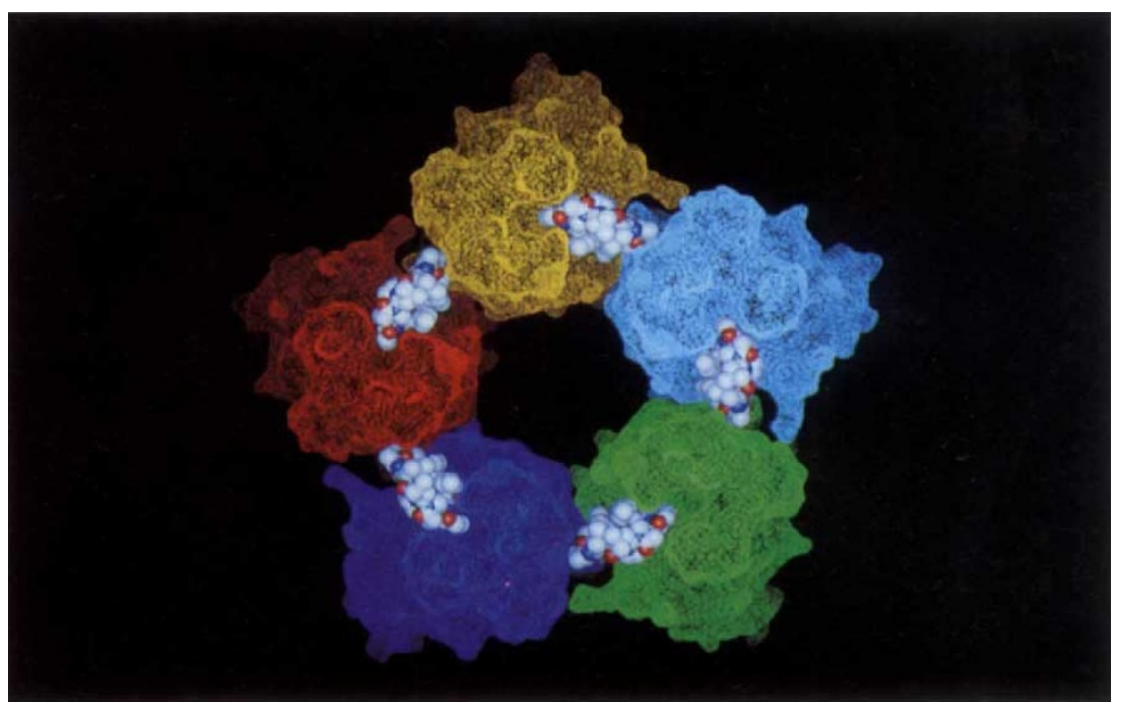

THE immunosuppressive drug cyclosporin A (CsA), a peptide fungal product that has been used for over a decade to prevent rejection of organ transplants, works by binding to members of the family of cyclophilin proteins. The drug-receptor complex mediates its immunosuppressive effect by interfering with the T-cell signal-transduction cascade, thereby preventing T-cell activation, a critical step in the immune response that causes transplant rejection. In investigating the $X$-ray structure of crystalline cyclophilin-cyclosporin A complexes, G. Pflügl et al. found the remarkable pentameric supermolecule shown above (cyclosporin, solid spheres; molecular surfaces of the five different coloured cyclophilin molecules shown as shrunken polygons using the program VIEW, M. Sanner, Sandoz). As the authors show on page $\mathbf{9 1}$ of this issue, these pentamers associate in pairs, sitting on top of one another, to form a ten-membered disk-like structure; the 'decameric sandwich'. The secondary structure elements of the cyclophilin A molecules, the $\beta$-sheets and $\alpha$-helices, are on the upper and lower faces of the disk and the CsA-binding loops and ten CsA molecules are sandwiched between them (the picture illustrates the inner face of one half of the sandwich). The biological significance of the pentamer and the decameric sandwich is as yet unclear, but there are hints that they may be more than an artefact of the crystallization process. The $X$-ray structure, as well as revealing the intriguing supramolecular organization of the cyclophilin-cyclosporin complex, sheds further light on details of the interaction of the peptide drug and its receptor, independently analysed with NMR by $Y$. Thériault et al. on page 88 . This information will be of great use in the design of CsA analogues.

G.R.

systems are limited, implicitly, to the range of stimuli considered, but this work makes clear the potential to jump out of such a system. At least temporarily, that is: for a jump out of an ESS system to be maintained, there must be an advantage to both the signaller and the receiver. Perhaps tellingly, the genetic algorithm's biases were for supernormal stimuli - it preferred bigger or longer instantiations of the stimuli which it had evolved to discriminate - rather than intermediate or qualitatively different forms. It is still early, but these results combined with the large number of selective forces acting on signalling systems (for example, most discrimination systems will be exposed to more than two forms) may greatly reduce the number and form of hidden biases.

Amotz Zahavi ${ }^{6}$ (University of Tel Aviv) carried a remarkable idea out of Israel and into the rest of evolutionary biology in the middle 1970 s, an idea that after a short period in the wilderness has now gained wide acceptance. Zahavi's idea was that when signals are used to persuade, as in threats or sexual attraction, signalling systems will evolve around costly, wasteful signals. Thus, the 'stot' of a Thompson's gazelle while being chased by a predator - as it runs it jumps up into the air holding its legs out straight - is an utterly wasteful, frivolous and even reckless thing to do. But this very wastefulness, ironically, assures the predator of the gazelle's ability to flee: stotting is an 'honest' signal because only the best quality gazelles can afford to 'handicap' themselves by stotting (see ref. 7). Similar logic has been applied to explain a diverse set of animal signals, and may help to explain why some people spend as much on a wristwatch as most of us would spend on a new car. Theoretical models of the handicap principle $e^{8-10}$ have assumed implicitly that receivers can perceive signals - such as the height of a stot - without error. New theoreti- 